\title{
The Costs of Emotional Attention: Affective Processing Inhibits Subsequent Lexico-semantic Analysis
}

\author{
Niklas Ihssen $^{1}$, Sabine Heim ${ }^{1}$, and Andreas Keil ${ }^{2}$
}

\begin{abstract}
The human brain has evolved to process motivationally relevant information in an optimized manner. The perceptual benefit for emotionally arousing material, termed motivated attention, is indexed by electrocortical amplification at various levels of stimulus analysis. An outstanding issue, particularly on a neuronal level, refers to whether and how perceptual enhancement for arousing signals translates into modified processing of information presented in temporal or spatial proximity to the affective cue. The present studies aimed to examine facilitation and interference effects of task-irrelevant emotional pictures on subsequent word identification. In the context of forced-choice lexical decision tasks, pictures varying in hedonic valence and emotional arousal preceded word/ pseudoword targets. Across measures and experiments, higharousing compared to low-arousing pictures were associated with impaired processing of word targets. Arousing pleasant
\end{abstract}

and unpleasant pictures prolonged word reaction times irrespective of stimulus-onset asynchrony (80 msec, $200 \mathrm{msec}$, $440 \mathrm{msec}$ ) and salient semantic category differences (e.g., erotica vs. mutilation pictures). On a neuronal level, interference was reflected in reduced N1 responses (204-264 msec) to both target types. Paralleling behavioral effects, suppression of the late positivity (404-704 msec) was more pronounced for word compared to pseudoword targets. Regional source modeling indicated that early reduction effects originated from inhibited cortical activity in posterior areas of the left inferior temporal cortex associated with orthographic processing. Modeling of later reduction effects argues for interference in distributed semantic networks comprising left anterior temporal and parietal sources. Thus, affective processing interferes with subsequent lexico-semantic analysis along the ventral stream.

\section{INTRODUCTION}

In a complex environment, adaptive behavior requires rapid extraction of critical sensory information. An observer must distinguish potential threats from potential benefits in order to optimize the behavioral outcome (Öhman, Flykt, \& Lundqvist, 2000). This capability has been theoretically related to evolutionary old emotional systems mediating perception and action in motivationally significant situations (Lang, Bradley, \& Cuthbert, 1997). In daily life, this is often reflected in behavioral tendencies of approaching versus avoiding stimuli. Laboratory experiments usually rely on symbolic stimuli such as pictures, movies, or words to systematically study aspects of emotional processing (e.g., Bradley et al., 2003). This line of research continues to accrue evidence showing that the human brain processes emotionally arousing information in an optimized manner (e.g., Phelps, Ling, \& Carrasco, 2006).

\section{Perceptual Enhancement for Emotional Information}

There are two key findings in electrophysiological research on visual processing of emotionally arousing

${ }^{1}$ University of Konstanz, Germany, ${ }^{2}$ University of Florida

stimuli, which have been replicated consistently: (i) Viewing emotional pictures was related to enhancement of a late positive component ( $>300$ msec poststimulus) of the event-related potential (ERP; Keil et al., 2002; Cuthbert, Schupp, Bradley, Birbaumer, \& Lang, 2000; Cacioppo, Crites, Berntson, \& Coles, 1993). (ii) An earlier negative amplitude shift over posterior scalp regions in the time segment between 150 and $250 \mathrm{msec}$ also varied as a function of emotionally arousing stimuli (Schupp, Junghöfer, Weike, \& Hamm, 2003; Junghöfer, Bradley, Elbert, \& Lang, 2001; Keil et al., 2001). This early ERP modulation, sometimes termed early posterior negativity (EPN), is thought to reflect a fast discrimination mechanism, which selects motivationally relevant information for further processing (Schupp et al., 2003). In analogy to so-called sensory gain accounts of volitional selective attention (e.g., Hillyard, Vogel, \& Luck, 1998), arousing stimuli are assumed to benefit from optimized sensory encoding (Schupp et al., 2003). Enhanced late positive potentials have been proposed to reflect increased attentional allocation and continued analysis of relevant stimuli at higher-order and postsensory stages of stimulus processing (Cuthbert et al., 2000). These two key findings can theoretically be linked to the concept of motivated attention, which posits that processing resources are automatically allocated to visual objects indexed as motivationally significant (Lang et al., 1997). In the same 
vein, emotional stimuli are identified with higher accuracy than neutral items, for example, in an attentional blink design (Keil, Ihssen, \& Heim, 2006; Keil \& Ihssen, 2004; Anderson \& Phelps, 2001) or a choice-reaction task (Zeelenberg, Wagenmakers, \& Rotteveel, 2006). The view of emotion guiding attentional resources and facilitating perceptual encoding is also corroborated by hemodynamic brain imaging studies. For instance, increased regional blood flow in occipital and inferior temporal visual cortices was found in response to emotionally arousing compared to neutral pictures (Junghöfer et al., 2006; Sabatinelli, Bradley, Fitzsimmons, \& Lang, 2005).

In the work outlined so far, stimuli varying in emotional intensity were presented as single exemplars in an isolated fashion. An interesting but less frequently addressed question refers to how motivated attention might affect encoding of information presented concurrently or in temporal proximity to the emotional stimulus. The present studies sought to explore consequences of motivated attention for neutral word stimuli presented shortly after an emotionally arousing picture. As described below, the fate of such stimuli can be predicted in two different ways.

\section{Resource Capture by Emotional Events and Affective Interference}

Numerous behavioral studies, in which emotional words or pictures served as distractors for a concurrent cognitive task, suggest that motivated attention interferes with competing processes. In emotional Stroop paradigms, for example, color naming latencies are slowed for words having aversive compared to neutral content (e.g., MacKay et al., 2004; Pratto \& John, 1991). This points to the capability of emotional events to automatically capture attentional resources. Detrimental capture effects have also been shown for neutral information presented with a temporal gap between the target and the emotional distractor. Foveally presented, task-irrelevant threat words slowed lexical decisions to neutral probe words at a stimulus-onset asynchrony (SOA) of $300 \mathrm{msec}$ (Calvo \& Castillo, 2005). Similarly, the detection of rotated landscape/architecture pictures amidst a rapid serial visual presentation (RSVP) stream is impaired when the targets are preceded by a negative compared to a neutral distractor (Most, Chun, Widders, \& Zald, 2005) within a time window of 200 msec.

These findings indicate that perceptual enhancement effects are restricted to the emotional stimulus and entail impaired processing of concurrent events. Electrocortical studies of affective interference, however, are rare. One line of research analyzed the P300 to acoustic startle probes as an indicator of resource deployment by concurrently presented emotional background stimuli (e.g., Schupp, Cuthbert, et al., 2004; Cuthbert, Schupp, Bradley, McManis, \& Lang, 1998). These authors reported a reduction of probe $\mathrm{P} 300$ amplitudes when view- ing arousing (pleasant and unpleasant) compared to nonarousing scenes. Probe stimuli were typically presented as late as 2.5-4.5 sec following picture onset (Schupp, Cuthbert, et al., 2004), so that these studies did not shed light on the short-term dynamics of emotional interference.

\section{An Emotion-induced Mode of Facilitated Processing?}

In addition to the described interference effects, a different prediction might be inferred from recent work by Phelps et al. (2006). Using centrally presented stimuli displayed at an SOA of $125 \mathrm{msec}$, they found that emotional events can facilitate subsequent visual processing. In an orientation discrimination task, contrast thresholds were lower for grating patterns presented after fearful faces compared to gratings following neutral faces. Phelps and colleagues concluded that "emotion facilitates early vision: People actually see better in the presence of emotional stimuli" (p. 298). Such transient but nonselective facilitation elicited by motivationally relevant stimuli might also be predicted from electrophysiological and behavioral work manipulating the distribution of visual spatial attention. For instance, Pourtois, Grandjean, Sander, and Vuilleumier (2004) presented pairs of faces in the left and right visual hemifield. One face showed a fearful and the other a neutral expression. With SOAs varying between 200 and $400 \mathrm{msec}$, the face pairs were followed by unilateral bars with either a horizontal or vertical orientation. Increased P1 amplitudes and faster discrimination responses to bars replacing fearful versus neutral faces indicated that fear cues facilitated processing of subsequent stimuli at their spatial location. In the context of event-related functional magnetic resonance imaging, such facilitation effects were demonstrated for target stimuli following cues with acquired emotional relevance (Armony \& Dolan, 2002). It is conceivable that the detection of any motivationally relevant event induces a transient attentional response, which temporarily tunes the brain for efficient encoding of subsequent sensory input. This implies that a quick glimpse on emotionally arousing scenes might shift the perceptual system to a facilitated processing mode, being unspecific with regard to all incoming information.

\section{The Present Studies}

The studies reviewed above have provided evidence for the capability of emotional stimuli to facilitate processing of temporally adjacent information, but also to impair subsequent processing. Thus, the question arises: Which variables mediate the divergent outcomes of facilitation versus interference as a consequence of affective processing? Importantly, differences related to emotional intensity/arousal and affective stimulus types (e.g., faces versus pictures) may contribute to the pattern of findings. 
Further, it is conceivable that the temporal distance or complexity of the primary task plays a key role in eliciting behavioral costs versus benefits. Here, we used a lexical decision as the primary task, which was preceded by taskirrelevant emotional pictures. This setup aimed to be sensitive to the influence of arousing material on higherorder processing as is the case with lexico-semantic analysis (Binder et al., 2003). Linguistic, as opposed to picture targets, reduce variance related to physical properties. Compared to simple visual stimuli (e.g., geometric shapes), they provide an opportunity for using a high number of stimulus exemplars, thus tapping more directly processes related to identification of new information. Affective pictures were used as precedent stimuli as they allowed us to maximize differences in emotional intensity of the irrelevant stimuli, as compared to word or face stimuli.

In Experiment 1, we employed a temporal interval of 200 msec between picture and target, which has been recently shown to provoke reliable emotion effects on subsequent target processing (Most et al., 2005). Behavioral and electrocortical dynamics were recorded while participants responded to word/pseudoword targets preceded by high-arousing pleasant pictures (erotica or sports), high-arousing unpleasant pictures (attack or mutilation), or low-arousing neutral pictures (neutral persons). Thus, Experiment 1 was intended (i) to explore the occurrence of behavioral facilitation/interference elicited by briefly presented arousing pictures within the context of lexico-semantic processing and (ii) to track electrocortical correlates of such effects at high temporal resolution. Electrophysiological measures at high temporal accuracy are particularly advantageous because interference effects may differentially affect subsequent processing, depending on the time range and brain processes involved.

Using the same stimuli as in the first study, Experiment 2 tested the possibility that the direction of behavioral emotion effects (facilitation versus interference) depended on the temporal interval between picture and target. As suggested by the findings of Phelps et al. (2006), transient facilitation may emerge within a narrow time window after offset of the emotional stimulus while later processes are suppressed. To test this hypothesis, picture-target pairs were presented at varying SOAs (80 msec, $200 \mathrm{msec}, 440 \mathrm{msec}$ ).

Experiment 3 examined the role of emotional arousal versus hedonic valence in the present paradigm. In particular, we sought to rule out that response modulation was driven by salient differences related to semantic picture categories (e.g., erotica vs. sports scenes) and not by variations in emotional arousal. To this end, responses to low-arousing pictures were contrasted with responses to high-arousing items having similar valence scores. In addition, postexperimental affective picture ratings were invited to predict reaction time (RT) modulation both on an individual subject and a single-item level.

\section{METHODS}

\section{Experiment 1}

\section{Participants}

Twenty-two healthy university students (11 women) with a mean age of 23.0 years (standard deviation $[S D]=5.9$ ) gave their informed consent to participate. As in the subsequent experiments, all participants were native speakers of German and reported normal or correctedto-normal vision. Twenty-one participants were righthanded, having a laterality quotient of $\geq 70$ in the Edinburgh Handedness Inventory (Oldfield, 1971). One subject was ambidextrous, but was retained in the sample, as she did not show any signs of outlying ERP activity. In all three experiments, participants received course credits or a financial bonus of 7.50 Euros.

\section{Stimuli}

Verbal stimuli comprised neutral German verbs (e.g., "beantworten" = to reply; "vorschlagen" = to propose) and pronounceable, arbitrary pseudowords (e.g., "beglinfen," "vorstelmen"). Selection of verbs was based on a pre-experimental rating study which used a paperand-pencil version of the 9-point-scale Self-Assessment Manikin (SAM; Bradley \& Lang, 1994). We obtained ratings for 160 verbs on the dimensions of emotional valence and arousal from 80 adults (mean age $=24.9$ years, $S D=$ 5.7, 38 women). Whereas judgments of hedonic valence indicate whether a stimulus is perceived as appetitive, neutral, or aversive, judgments of arousal reflect the intensity of motivational activation (Lang et al., 1997). We selected 90 verbs with low arousal (mean $=3.8, S D=$ 0.6 ) and neutral valence (mean $=5.4, S D=0.4$ ) scores. Using this set, 90 pseudowords were generated by permuting syllables and letters within or between verb stimuli. Pseudowords and words were matched for item length, initial letters, prefixes, and endings.

Pictures were taken from a pre-experimental study using a computerized version of the SAM. A group of 45 adults (mean age $=23.3$ years, $S D=4.2 ; 26$ women) judged 216 pictures from the International Affective Picture System (IAPS; Lang, Bradley, \& Cuthbert, 2005), the Graphics Suite CD (Micrografx, 1997), and various Web sites. Each stimulus displayed at least one human agent. Based on these ratings, 180 pictures were selected: (i) 60 high-arousing pleasant items including 30 erotica (mean valence $=6.6, S D=0.5$; mean arousal $=6.0, S D=$ $0.5)$ and 30 sports and adventure scenes (mean valence $=$ $6.5, S D=0.6$; mean arousal $=5.5, S D=0.4$ ); (ii) 60 lowarousing neutral pictures depicting, for instance, office activities (mean valence $=5.2, S D=0.4$; mean arousal $=$ $2.7, S D=0.4$ ); and (iii) 60 high-arousing unpleasant pictures showing 30 attack scenes (mean valence $=2.5$, $S D=0.4$; mean arousal $=6.3, S D=0.5$ ) and 30 exemplars of mutilated bodies or injured people (mean valence $=$ $1.5, S D=0.4$; mean arousal $=7.0, S D=0.6$ ). Within each 
affective category, pairs of stimuli were selected displaying equivalent content (e.g., two pictures showing windsurfing, two pictures showing people in roller coasters). This resulted in two semantically congruent sets A and B of stimuli, consisting each of 30 pleasant, 30 neutral, and 30 unpleasant pictures.

\section{Procedure}

Subjects were seated comfortably in an electrically shielded, sound-attenuated, and dimly lit recording chamber. They viewed the stimuli on a 19-in. computer screen at an average distance of $70 \mathrm{~cm}$. A chin rest helped to reduce movement artifacts and interindividual variability in terms of head position and visual angle. All stimuli appeared centrally against a gray background.

Participants performed five practice trials involving stimuli not used in the test trials. Each trial started with a $14.5 \times 20 \mathrm{~cm}$ picture appearing for $100 \mathrm{msec}$, followed by a random dot mask of equal size and duration. Insertion of a mask aimed to help separating electrocortical activation patterns evoked by picture versus word presentation. Verbal targets were shown in white color immediately after mask offset, resulting in a picture-target SOA of $200 \mathrm{msec}$. The targets were presented in 36-point Times New Roman font using lowercase letters. Participants were asked to indicate whether the target stimulus was a real word in German by pressing the left mouse button with their right index finger or a nonsense word by pressing the right button with their right middle finger. Button assignments were counterbalanced across subjects. Participants were asked to give their response as fast and accurate as possible within a time window of $2000 \mathrm{msec}$. Target presentation was terminated by the response. No feedback was provided. After an interval randomly varying between 2500 and $3500 \mathrm{msec}$ and displaying a central fixation cross, the next trial started. The experimental session was divided into two runs separated by a short break. During each run, each of the 90 words and 90 pseudowords was presented once in random order, resulting in 360 trials for the whole session. In the first run, word targets were randomly combined with pictures from set A and pseudowords with those from set $\mathrm{B}$ (see above). The assignment of words/pseudowords to the picture sets was reversed in the second run. The order of set assignment was counterbalanced across participants.

\section{Analysis of Behavioral Data}

For each participant and Picture $\times$ Target combination, the mean RT and error rate was calculated. RTs were analyzed for correct responses occurring in a time segment of 200 to $2500 \mathrm{msec}$ following target onset.
We selected a specific cutoff value (2500 msec) for dealing with RT outliers instead of applying thresholds on the basis of individual RT distributions (SD trimming, Windzorizing, etc.). With regard to statistical power, fixed thresholds have been proven equally or even superior, compared to variable criteria (Ratcliff, 1993). Error rate was computed as the percentage of trials with misses, wrong responses, and responses occurring earlier than $200 \mathrm{msec}$ after target onset. The two dependent variables entered separate analyses of variance (ANOVAs) crossing the within-subjects factors picture category (erotica, sports, neutral, attack, mutilation) and target type (word, pseudoword). Significant RT effects including picture category were followed by trend analyses. We expected a quadratic trend exhibiting a parabolic pattern of mean RTs with disproportionately strong modulation for the erotica and mutilation subcategories (i.e., erotica $>$ sports $>$ neutral $<$ attack $<$ mutilation). This prediction is in line with recent notions that affective modulation of perception is most evident for stimuli with high evolutionary significance (Schupp, Flaisch, Stockburger, \& Junghöfer, 2006).

\section{EEG Recording and Preprocessing}

The electroencephalogram (EEG) was recorded continuously from 129 channels using an Electrical Geodesics (EGI) system. Data were digitized at a rate of $500 \mathrm{~Hz}$ and were filtered on-line between 0.01 and $100 \mathrm{~Hz}$. As recommended for the EGI high input-impedance amplifier, impedances were kept below $50 \mathrm{k} \Omega$. The vertex sensor (electrode $\mathrm{Cz}$ ) was used as recording reference. Channels with bad signal throughout the whole recording session (mean $=4.5$ channels, $S D=2.2$ ) were interpolated off-line using the spline interpolation method (Perrin, Pernier, Bertrand, \& Echallier, 1989) of the Besa software. An eye artifact correction was performed on the raw data applying the algorithm implemented in Besa (Berg \& Scherg, 1994b). Epochs were then extracted from $500 \mathrm{msec}$ before until $900 \mathrm{msec}$ after target onset. Based on visual inspection, the remaining epochs being afflicted with artifacts were rejected (on average, 3.7\% per condition). Using the average reference, artifact-free epochs were then averaged separately for the 10 combinations of picture category (erotica, sports, neutral, attack, mutilation) and target type (word, pseudoword). In order to achieve comparable signal-to-noise ratios across combinations, we randomly drew $50 \%$ of the trials in the neutral picture-word/pseudoword conditions. Only those trials with correct responses to targets were considered. Artifact and error trial exclusion resulted in a mean of $27.5(S D=0.8)$ epochs per Picture $\times$ Target combination and subject. Averaged data were filtered between 1 and $30 \mathrm{~Hz}$, and the mean of a 200-msec segment prior to picture onset was subtracted as baseline. 


\section{ERP Analysis}

Statistical analysis of the three selected ERP deflectionspicture P2, target N1, and target late positivity (LP; see Results)—was performed using regional means. Sensor clusters were selected according to the spatial focus of the respective component in the grand-average topographical maps. Defining regional means instead of using single sensor information has been reported to enhance the reliability of the estimate used as dependent variable (Keil, Stolarova, Heim, Gruber, \& Müller, 2003). For analysis of the picture P2 and target N1, a symmetrical pair of sensor clusters was chosen comprising nine electrodes over the left and nine electrodes over the right occipital cortex (left hemisphere electrodes 59, 65, 66, 69, 70, 71, 72, 74, 75; right hemisphere electrodes 77 , $83,84,85,89,90,91,92,95)$. For the target LP component, parieto-central regional means were formed each containing nine electrodes over the left hemisphere (32, $38,43,48,52,53,54,60,61)$ and nine electrodes over the right hemisphere $(79,80,81,86,87,88,93,94,99)$. Figure 1 (left panel) shows the selected sensor clusters with respect to sites of the international 10-20 system. For each regional mean, peak latencies and mean amplitudes were examined for each participant. Peak latency of the picture P2 component was determined within a time range from 120 to $200 \mathrm{msec}$ after picture onset (i.e., -80 to 0 msec relative to target onset). Target N1 latency was scored between 184 and $284 \mathrm{msec}$, and LP latency between 412 and 712 msec after target onset. Amplitudes for the picture P2 and target N1 were averaged across a 60-msec segment around an individual's peak. A longer segment $(300 \mathrm{msec})$ was used around individual peaks of the LP. Mean amplitudes and peak latencies were submitted to ANOVAs having factors of picture category (erotica, sports, neutral, attack, mutilation), target type (word, pseudoword), and hemisphere (right, left). Similar to behavioral analyses, amplitude differences between picture categories were further evaluated by quadratic trend tests.

\section{Source Analysis}

Given the goal of the present study to examine effects of emotional processing on subsequent lexico-semantic analysis, we aimed to increase the spatial and temporal specificity of our analysis by means of source modeling. To specifically highlight the temporal dynamics within and across different neuroanatomic structures, we employed discrete regional sources linked to landmark regions of language processing (Cohen \& Dehaene, 2004;

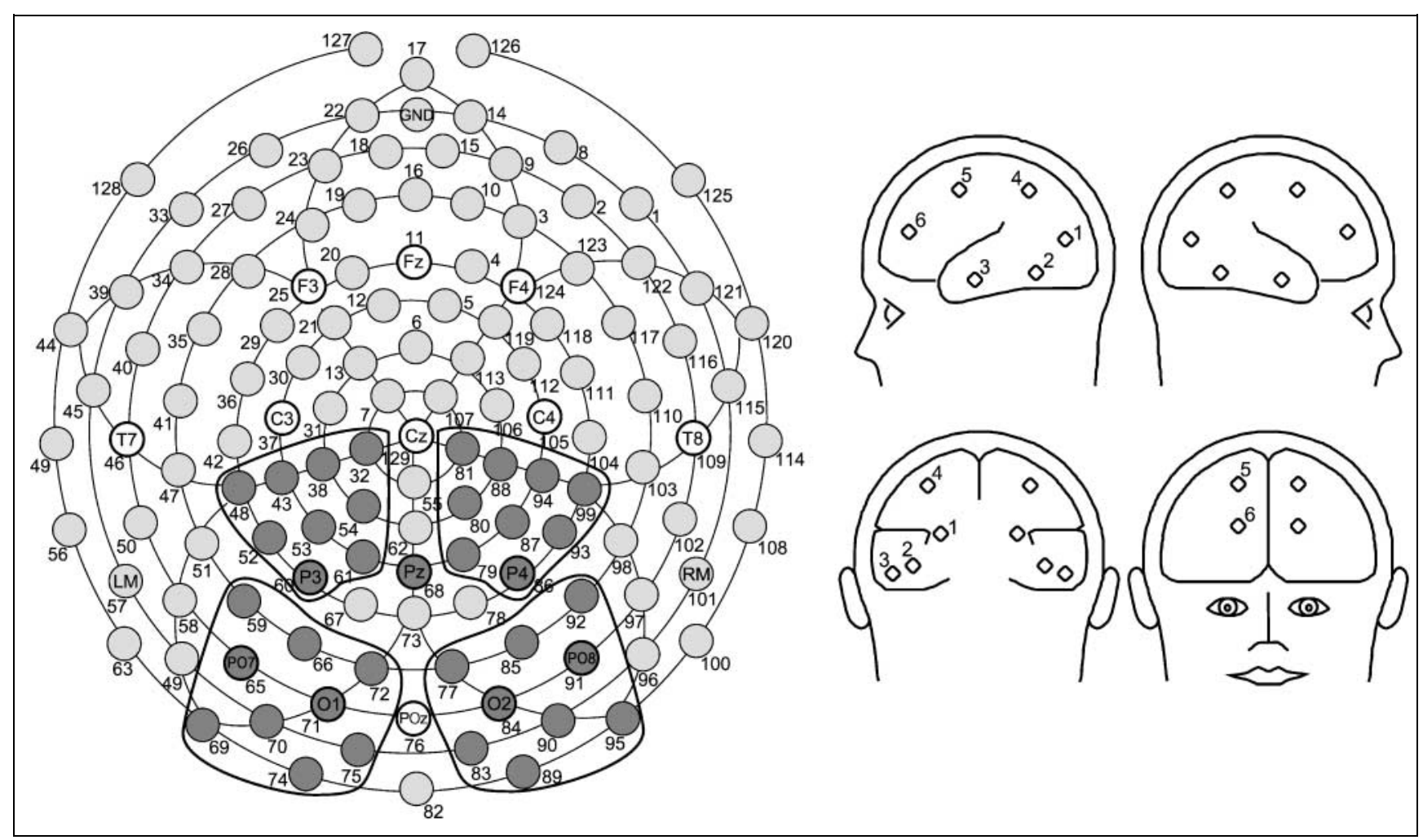

Figure 1. (Left) Layout of the electrode array used in Experiment 1. Shaded electrode sites in the marked clusters were selected to form regional means entering statistical analyses. The posterior sensor clusters were used to quantify picture P2 and target N1 components, the central clusters were chosen to quantify the target late positivity (LP). (Right) Regional source montage applied to single-subject ERPs. The model included 12 bilateral symmetric sources in occipital (labeled 1 in the left hemisphere), posterior inferior temporal (pIT, 2), anterior inferior temporal (aIT, 3), parietal (4), central (5), and frontal (6) cortices. This procedure aimed to track word processing proceeding along the ventral stream in the anterior direction with continued analysis. 
Hickok \& Poeppel, 2004). Regional sources do not aim to provide precise neuroanatomic localization, but represent the multielectrode ERP data in an anatomically meaningful low-dimensional space. This overcomes some of the problems associated with using voltage maps (Scherg, Ille, Bornfleth, \& Berg, 2002). As a key advantage, the interpretation of source current amplitude enhancement as increase in brain electric activity is possible. Regional sources are sensitive to current changes irrespective of their orientation in space and therefore capture electrocortical activity originating from a wider range of areas. It is important to ensure that activity is not misrepresented in an area far from the true underlying source. Here, we used source sensitivity maps implemented in the Besa software to measure whether the model dipoles were sensitive to local changes only. To calculate source sensitivity, the software models unit brain activity at different locations throughout the brain. Source sensitivity is defined as the fraction of power that is mapped on the selected source given the applied head model and regularization constant.

We seeded 12 symmetric bilateral sources in a fourshell ellipsoidal head model (Berg \& Scherg, 1994a). Positioning of regional dipoles drew on recent theories about the functional neuroanatomy of language (Cohen \& Dehaene, 2004; Hickok \& Poeppel, 2004; Fernandez et al., 2001), which posit that lexico-semantic analysis of verbal material is related to activation of the ventral processing system (Ungerleider \& Mishkin, 1982). During visual word recognition, intracranial recordings in the inferior temporal cortex have revealed a directed processing stream proceeding in anterior direction with continued stimulus analysis (Fernandez et al., 2001). Left posterior inferior temporal areas also constitute a core element of theories which argue that visual word form activates specialized "word form areas" in the left fusiform gyrus (e.g., Cohen \& Dehaene, 2004). The source montage as used here is illustrated in Figure 1 (right panel). It included symmetric bilateral dipoles in the occipital cortex as well as the posterior and anterior inferior temporal lobe (pIT and aIT). The left pIT was seeded according to the Talairach coordinates proposed "as the peak of the visual word form area" (McCandliss, Cohen, \& Dehaene, 2003, p. 294) based on reviews of over 25 imaging experiments. The aIT was seeded to pick up ventral stream activity more rostrally. Further, the model included sources in parietal, central, and frontal cortices to control for activity outside the ventral system and to ensure sensitivity of each source for a particular brain region. Occipital, central, and frontal sources were equidistant to the midline; frontal and central sources were seeded according to equal $x$ locations. Talairach coordinates of the regional dipoles are listed in Table 1 .

The 12-source model was applied to individual ERPs. To reduce the number of comparisons, statistical evalu-
Table 1. Talairach Coordinates of the Regional Sources Used to Model ERP Data

\begin{tabular}{lcrr}
\hline & $x$ & $y$ & \multicolumn{1}{c}{$z$} \\
\hline Occipital & -25.0 (right 25) & -80.0 & 9.0 \\
Posterior inferior temporal & -43.0 (right 43.0) & -54.0 & -12.0 \\
Anterior inferior temporal & -55.3 (right 55.3) & -2.9 & -12.0 \\
Parietal & -33.3 (right 33.3) & -54.8 & 46.1 \\
Central & -19.2 (right 19.2) & 0.9 & 52.0 \\
Frontal & -19.2 (right 19.2) & 45.0 & 27.1 \\
\hline
\end{tabular}

Coordinates refer to sources in the left hemisphere. Right hemispheric sources were seeded symmetrically to the left sources, thus yielding deviant coordinates only on the $x$-dimension.

ation of source intensity was restricted to three levels of picture category (pleasant, neutral, unpleasant). For each participant, mean source activity in the N1 window was calculated between 204 and 264 msec after target onset, representing a 60-msec segment around N1 mean latency (234 msec). Mean source intensity in the LP window was obtained from 404 to 704 msec after target onset, representing a 300-msec segment around LP mean latency (554 msec). For each source and target type (word, pseudoword), mean intensity values were submitted to ANOVAs using picture category as withinsubject factor. Due to the augmented number of comparisons, the alpha level was adjusted to $1 \%$. Significant effects were analyzed by means of Newman-Keuls tests.

\section{Experiment 2}

\section{Participants}

Twelve university students consented to participate in Experiment 2 (11 women; mean age $=22.2$ years, $S D=4.2$ )

\section{Stimuli}

Word/pseudoword targets were identical to Experiment 1. With regard to picture stimuli, three categories were used: erotica (high-arousing pleasant, 60 items), neutral persons (low-arousing, 60 items), and mutilation (higharousing unpleasant, 60 items) pictures. The same pictures as in Experiment 1 were employed plus additional items required for the erotica and mutilation category. Again, the entire picture set comprised two semantically congruent subsets assigned to word versus pseudoword targets in a counterbalanced manner across participants.

\section{Procedure}

Experimental setting, physical stimulus properties, and response modalities were the same as in Experiment 1. Targets followed pictures at three different SOAs (80 msec, $200 \mathrm{msec}, 440 \mathrm{msec}$ ). In the short SOA condition, targets 
were presented immediately after picture offset. In the 200- and 440-msec conditions, pictures were shown for $100 \mathrm{msec}$, followed by a blank screen with a duration of $100 \mathrm{msec}$ and $340 \mathrm{msec}$, respectively. Each of the 180 pictures (90 assigned to words, 90 assigned to pseudowords, see above) was shown once at each SOA resulting in 540 trials, which were presented in random order and included two breaks. Again, picture-word pairs were separated by an intertrial interval randomly varying between 2500 and 3500 msec. After the lexical decision task, participants rated the pictures using the computerized SAM version described above. To reduce the length of the experiment, only those pictures were judged that had preceded word targets.

\section{Data Analysis}

RTs and error rates were calculated as specified in Experiment 1. The two measures were submitted to repeated measures ANOVA with the factors SOA $(80 \mathrm{msec}$, $200 \mathrm{msec}, 440 \mathrm{msec}$ ), picture category (pleasant, neutral, unpleasant), and target category (word, pseudoword). Mean picture ratings of each participant were analyzed by one-factorial ANOVA using picture category as within-subject factor. Significant effects were followed by Newman-Keuls tests.

\section{Experiment 3}

\section{Participants}

Data were collected from 16 university students (14 women) having a mean age of $21.3(S D=2.7)$ years. Rating data from one subject were excluded because of lack of compliance.

\section{Stimuli}

Verbal targets were the same as in the previous experiments. Pictures were 180 items drawn from the IAPS (Lang et al., 2005). Picture selection aimed at building five stimulus categories (low-arousing pleasant [PL], high-arousing pleasant $[\mathrm{PH}]$, low-arousing neutral [NL], low-arousing unpleasant [UL], high-arousing unpleasant $[\mathrm{UH}] ; n=36$ items in each category) that were distinctive with regard to normative affective ratings but semantically heterogeneous. For instance, stimuli included both low-arousing and high-arousing erotica, or lowarousing and high-arousing attack scenes. According to the normative IAPS ratings, valence scores ranged from 6.1 to 8.2 for PL, from 5.6 to 8.0 for $\mathrm{PH}$, from 4.2 to 5.5 for NL, from 1.8 to 3.0 for UL, and from 1.3 to 3.8 for $\mathrm{UH}$ pictures. Arousal was between 2.9 and 4.9 for PL, between 5.3 and 7.2 for $\mathrm{PH}$, between 2.4 and 4.2 for NL, between 3.9 and 5.8 for UL, and 5.8 and 7.3 for $\mathrm{UH}$ pictures. Words/pseudowords were assigned to pictures by using the procedure described in Experiment 1. All pictures were rated after the experiment.

\section{Procedure}

The procedure was identical to Experiment 1.

\section{Data Analysis}

Analysis of responses paralleled the approach employed in the previous experiments. RTs and error rates were analyzed by means of 5 (picture category: PL, PH, NL, $\mathrm{UL}, \mathrm{UH}) \times 2$ (target category: words vs. pseudowords) repeated measures ANOVA. A quadratic trend was fitted to the data (see Experiment 1), expecting a moderate RT increase for PL and UL pictures and a strong impairment for $\mathrm{PH}$ and $\mathrm{UH}$ items. Ratings were analyzed with ANOVA as specified in Experiment 2. In addition, a correlational analysis was performed. For each participant, mean differences were calculated between arousal ratings for emotional categories versus the neutral category, and these values were correlated with the respective RT differences. In addition to these by-subject analyses, we tested the predictive value of single pictures' arousal ratings with regard to modulatory effects on RTs. This by-item analysis was done by using single pictures as observations and correlating pictures' mean arousal ratings with mean RTs.

\section{RESULTS AND DISCUSSIONS}

\section{Experiment 1}

\section{Behavioral Data}

Consistent with previous work (Hauk, Davis, Ford, Pulvermüller, \& Marslen-Wilson, 2006), subjects responded more slowly to pseudoword compared to word targets $[F(1,21)=29.0, p<.001]$. As indicated by a significant main effect of picture category $[F(4,84)=$ $3.9, p<.01]$, and a significant interaction of Picture category $\times$ Target type $[F(4,84)=8.0, p<.001]$, response latencies were also modulated by emotional content of the preceding stimulus. As illustrated in Figure 2, word RTs followed a U-shaped function with elevated values, that is, slowed RTs, for pictures depicting erotic and mutilation scenes [quadratic trend, $F(1$, $21)=21.7, p<.001]$. We did not find such a quadratic trend for responses to pseudowords $(F<1.0)$. Similar to RT analysis, ANOVA on error rates showed a significant interaction between picture category and target type $[F(4,84)=6.1, p<.001]$. Post hoc tests (NewmanKeuls) revealed higher error rates for word responses preceded by mutilation versus neutral pictures $(p<.05)$. Error rates on pseudoword trials did not differentiate between neutral and emotional pictures ( $p s>.53)$.

Taken together, behavioral data indicate that lexicosemantic processing is impaired and not facilitated 
Figure 2. Experiment 1: (Left) Mean reaction times (RTs) as a function of target type and category of the preceding picture. (Right) Mean emotional arousal ratings of the presented pictures as obtained in a pre-experimental study $(1=$ low arousing, $9=$ high arousing). The dotted line connecting mean RTs to words reflects a quadratic trend. Error bars indicate standard errors (standard deviations for arousal ratings).

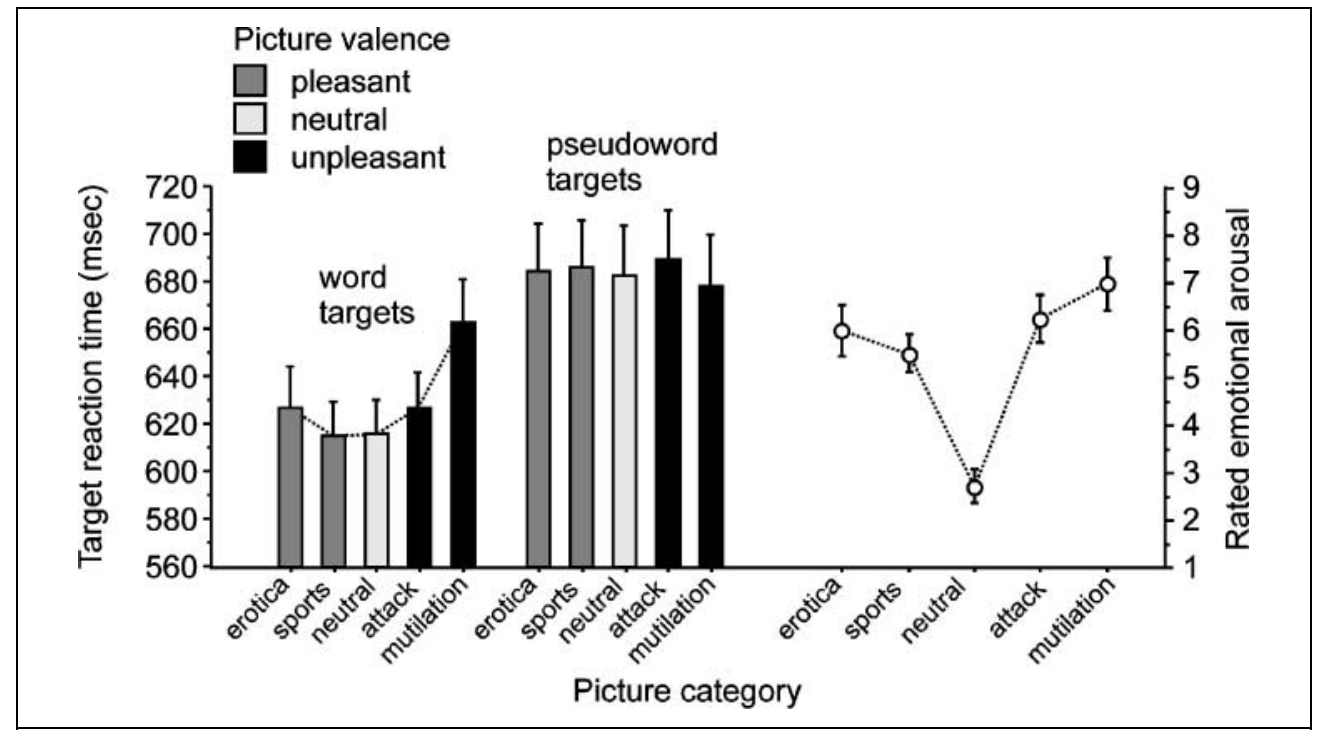

subsequent to information containing arousing appetitive or aversive content, such as erotic or mutilation scenes. Costs of processing these stimuli, however, are only evident for word but not for pseudoword responses. The question arises why arousing sports and attack pictures did not delay word responses (see Figure 2). We speculate that the small increase of subjectively rated arousal from sports to erotica, and from attack to mutilation, respectively (see Figure 2, right panel), underestimates the impact of pictures displaying content of high evolutionary significance (Schupp et al., 2006), such as sexuality or physical damage.

\section{Electrophysiological Data}

Over the posterior scalp, grand-average ERPs were characterized by a series of deflections reflecting the rapid sequence of visual events (i.e., picture-mask-target) within each trial (see Figure 3). These posterior components demonstrated polarity reversal over anterior electrodes. Three deflections were reliably detected in all participants: (i) a positive component peaking $160 \mathrm{msec}$ following picture onset, with a bilateral occipital maximum (picture P2); (ii) a bilateral occipito-temporal negativity reaching a maximum amplitude at about $230 \mathrm{msec}$ after word/pseudoword onset (target N1); and (iii) a late positivity over centro-parietal sensors extending from 400 to 700 msec post target (target LP). Deflections between picture P2 and target N1 were not analyzed as they likely reflected sensory responses to the mask (see Figure 3). Regarding target ERPs, we replicated the electrocortical pattern recently reported by Hauk et al. (2006). Using a forced-choice lexical decision task with English nouns, these authors observed a prominent occipital negativity and a centro-parietal positivity around 200 and $500 \mathrm{msec}$, respectively. ERP waveforms comprising the present picture P2 and target N1 at a representative left posterior electrode (PO7) are shown in Figure 4. Figure 6 depicts the target LP at a representative left central sensor.

Picture P2. Picture P2 amplitudes varied as a function of picture category $[F(4,84)=36.2, p<.001]$, whereas there were no effects of target type or hemisphere (all $F$ S $<1.1$ ). As can be seen in Figure 4, the P2 was more negative for emotional compared to neutral pictures. Trend analysis showed that across target types and hemispheres, negativity increased slightly from neutral to sports/attack pictures and strongly from neutral to erotica/mutilation pictures [quadratic trend: $F(1,21)=$ $59.2, p<.001]$. This finding replicates previous work showing early ERP negativity as a function of emotional intensity (Schupp et al., 2003) and suggests that subjects discriminated early (and prior to target onset) between different affective picture categories. Peak latencies were not sensitive to picture category $(F<1.0)$ or target type $(F<1.1)$.

Target N1. A significant main effect of target type $[F(1$, $21)=22.3, p<.001]$ indicated that $\mathrm{N} 1$ responses were smaller for words compared to pseudowords. N1 responses were also modulated by picture category $[F(4$, $84)=8.0, p<.001]$, reflecting reduced amplitudes for targets following arousing pictures. Trend analysis confirmed that across hemispheres and target types, erotic and mutilation pictures suppressed N1 responses markedly, whereas sports and attack scenes elicited no or only a small N1 reduction (see Figure 5) [quadratic trend: $F(1$, $21)=13.6, p<.01]$. This result corroborates the notion that the observed effects were driven by affectivesemantic factors and not by physical differences between the pleasant, neutral, and unpleasant stimulus categories. ANOVA on N1 peak latencies revealed that picture category also affected temporal aspects of processing $[F(4,84)=4.9, p<.01]$. Across target types and 
Figure 3. Experiment 1: Grand mean ERPs to word targets following pleasant (dashed line), neutral (solid line), and unpleasant (dotted line) pictures at a subset of electrodes corresponding to sites of the international 10-20 system. Time zero represents word target onset.

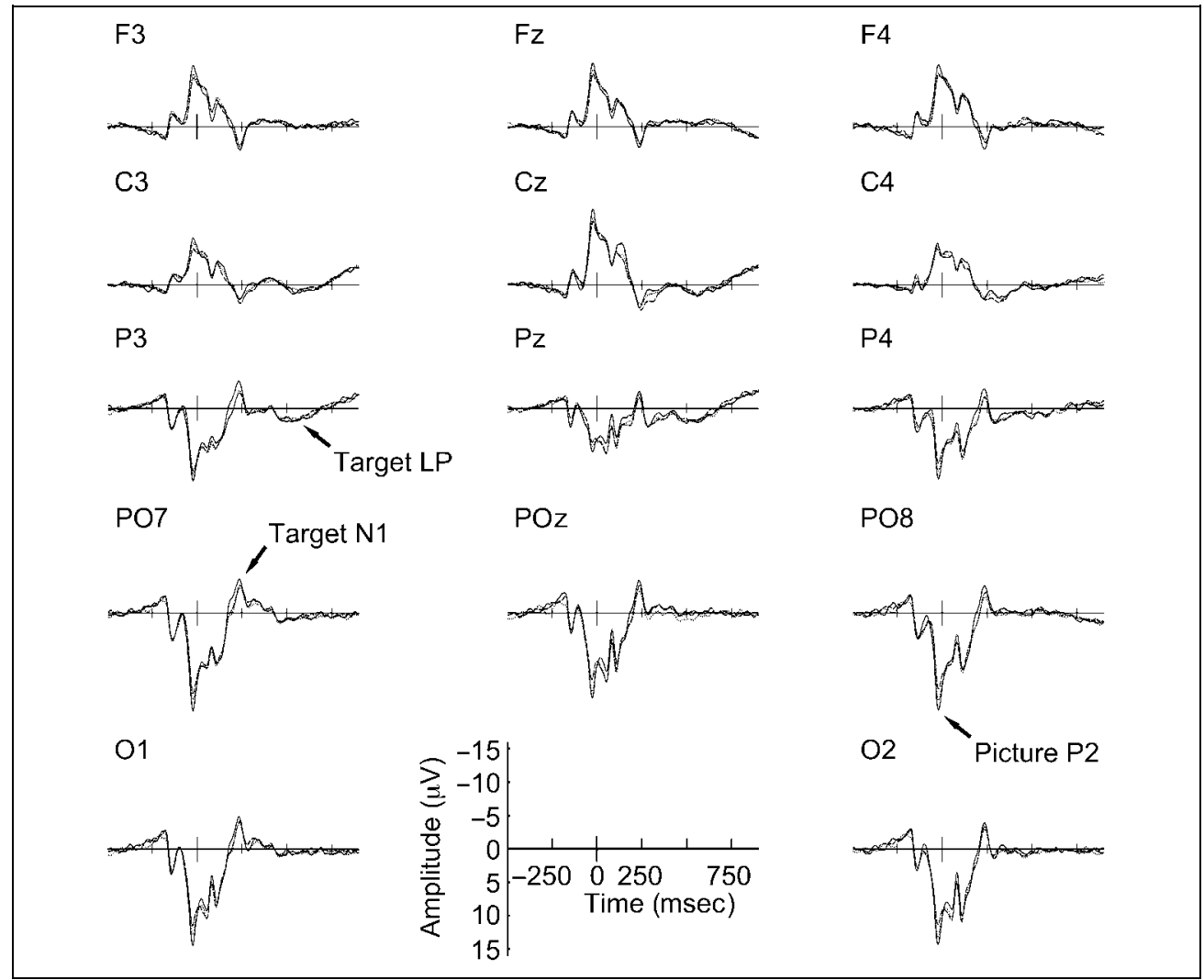

hemispheres, N1 maxima were clearly delayed for erotica (mean latency $=241 \mathrm{msec}, S E=2.7$ ) and mutilation (239 msec, $S E=2.3$ ) pictures, compared to neutral pictures (229 msec, $S E=2.2)$. Again, interference, namely, N1 latency increase, was less pronounced for sports $(235 \mathrm{msec}, S E=2.2)$ and attack $(234 \mathrm{msec}, S E=$ 1.9) pictures, resulting in a significant quadratic trend $[F(1,21)=9.0, p<.01]$. Notably, as opposed to the RT data, effects of emotion on the N1 were not sensitive to target lexicality showing reduced and delayed responses to words and pseudowords.
Target late positivity. ANOVA on LP amplitudes showed significant main effects of picture category $[F(4,84)=$ $3.9, p<.01]$ and target type $[F(1,21)=23.8, p<.001]$, as well as an interaction between these factors $[F(4,84)=$ 5.0, $p<.01]$. Hemisphere also affected LP amplitudes [main effect: $F(1,21)=4.6, p<.05$; Hemisphere $\times$ Target: $4.9, p<.05]$. As can be seen in Figure 6 , the LP was larger for words than pseudowords, and in the left hemisphere compared to the right hemisphere. Further, the difference between words and pseudowords was more pronounced in the left hemisphere. Trend
Figure 4. Experiment 1: Picture P2 and target N1 at a representative left occipital sensor (\#65, corresponding to PO7). Waveforms show grand mean ERPs in response to words (left) and pseudowords (right) following pleasant (dashed line), neutral (solid line), and unpleasant (dotted line) pictures. Shaded bars illustrate that picture $\mathrm{P} 2$ and target N1 were statistically analyzed by extracting mean amplitudes across a 60-msec time segment around individual subjects' peak latencies.

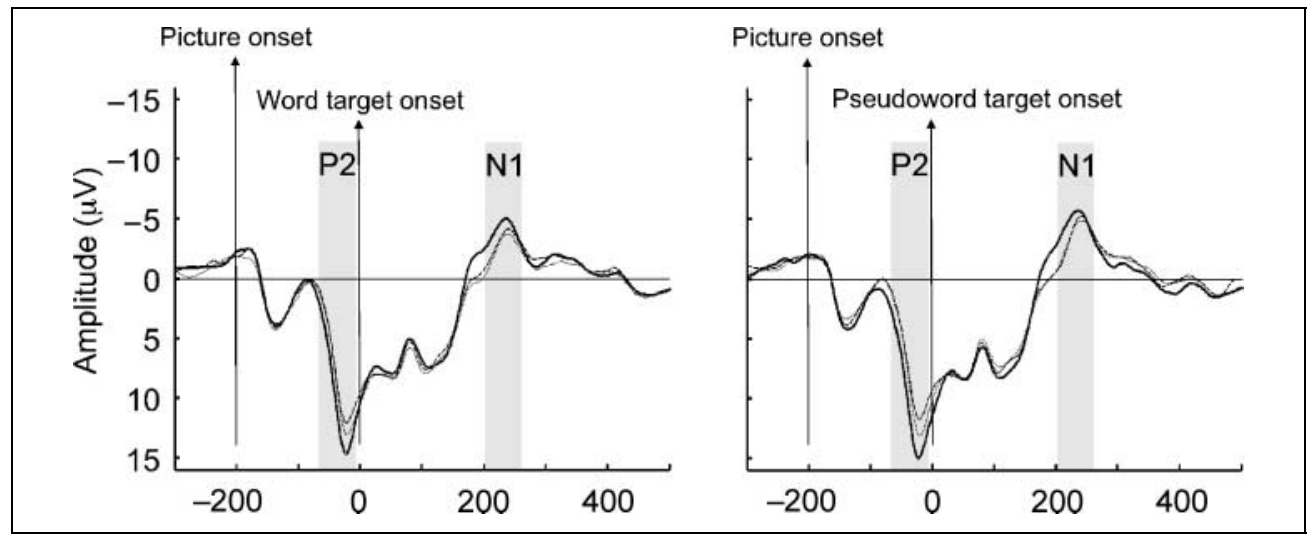


Figure 5. Experiment 1: Mean target N1 amplitudes as a function of target type and category of the preceding picture. For both targets (words and pseudowords), the N1 decreased with increasing emotional intensity of the preceding picture, irrespective of picture valence. Error bars represent standard errors.

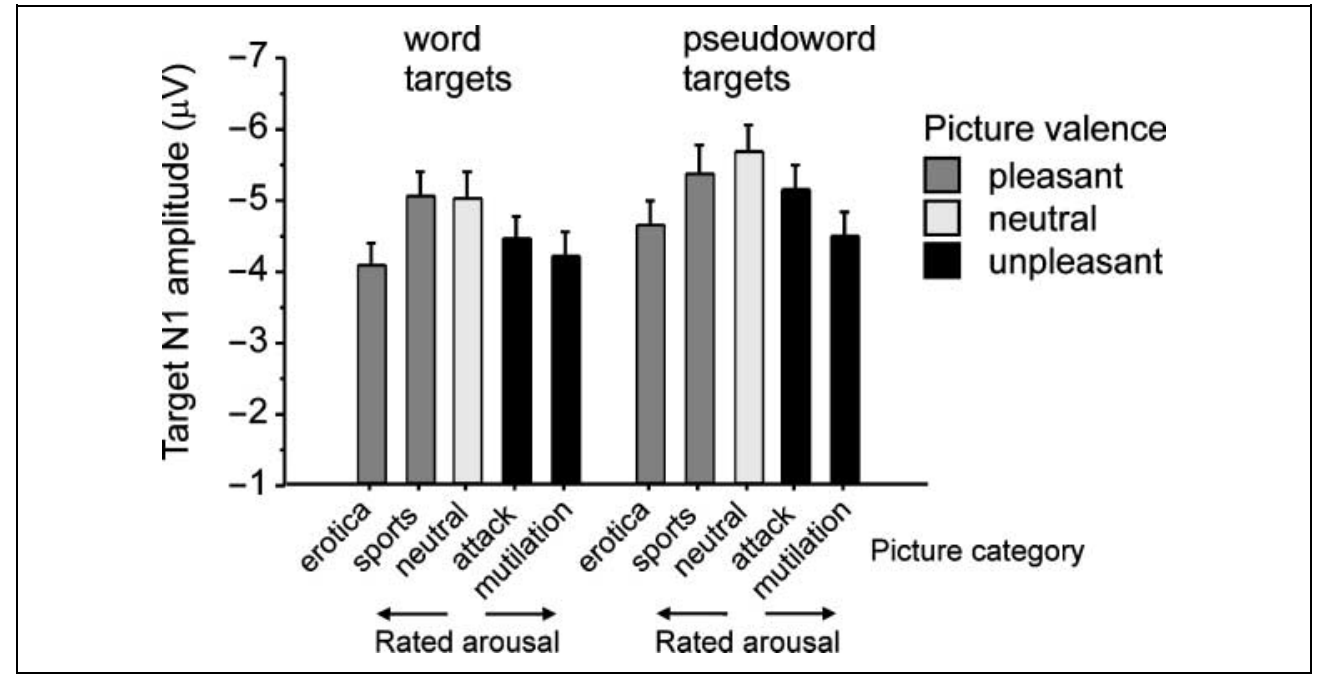

analyses testing the effects of picture category showed that emotional pictures reduced word LPs in the predicted manner in the left hemisphere [quadratic trend: $F(1,21)=4.9, p<.05]$, and in the right hemisphere $[F(1,21)=6.5, p<.05]$. The quadratic trend did not reach significance for pseudowords in either hemisphere [left: $F(1,21)=3.3, p=.08$, right: $F(1,21)=4.3, p=.05]$. That is, similar to the RT data, interference was more evident for word compared to pseudoword targets (Figure 7).

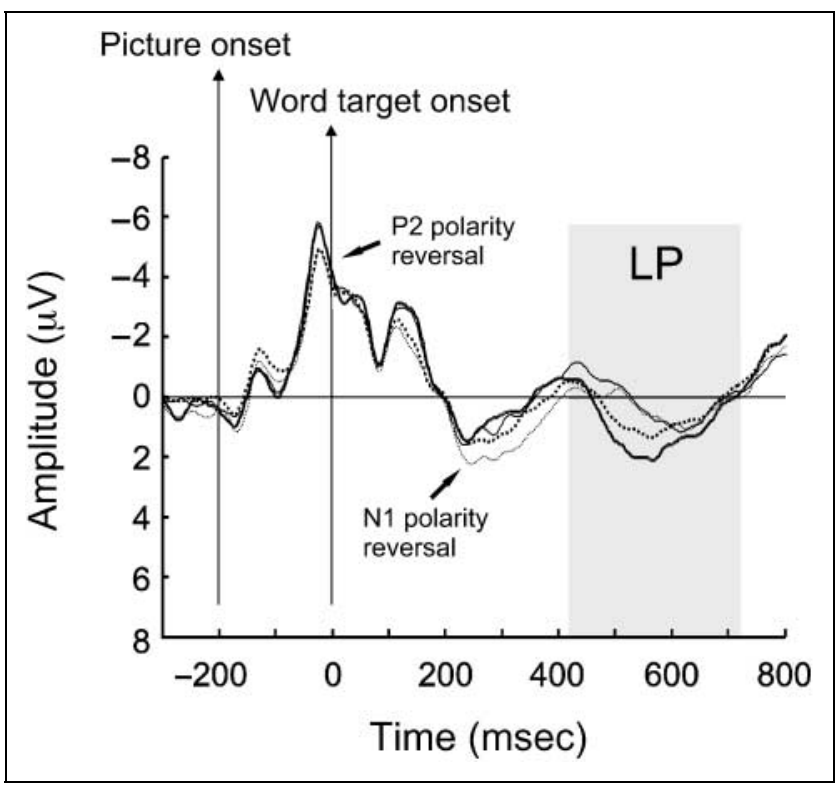

Figure 6. Experiment 1: Target LP at a representative left central sensor (\#38). Waveforms illustrate grand mean ERP responses to words following neutral pictures (thick solid line), to pseudowords following neutral pictures (thin solid line), to words following unpleasant pictures (thick dotted line), and to pseudowords following unpleasant pictures (thin dotted line). The shaded bar marks the 300-msec time window used to quantify LP mean amplitudes around individual subjects' LP peaks.
Peak latency analysis indicated that the LP reached maximum earlier for word (mean latency $=540 \mathrm{msec}$, $S E=5.2$ ) than for pseudoword targets (mean latency $=$ 564 msec, $S E=5.6)$ [target type: $F(1,21)=6.9, p<$ $.05]$. ANOVA also yielded significant interactions between picture category and target type $[F(4,84)=3.1$, $p<.05]$, and between picture category, target type, and hemisphere $[F(4,84)=3.8, p<.01]$. Trend analyses revealed a significant quadratic trend for word targets in the left $[F(1,21)=8.7, p<.01]$, but not in the right hemisphere $(F<1.0)$. For pseudowords, quadratic trends did not reach significance [left: $F<1.0$; right: $F(1,21)=3.9, p=.06]$. In contrast to the $\mathrm{N} 1$ delay, the emotion effect on LP maxima was reflected in shorter latencies for (left hemispheric) word responses following erotica $(517 \mathrm{msec}, S E=19.0)$ and mutilation (527 msec, $S E$ 14.0) pictures and slightly earlier peaks for word responses following sports $(554 \mathrm{msec}, S E=$ 9.6) and attack ( $546 \mathrm{msec}, S E=12.7)$ pictures, compared to the neutral category $(556 \mathrm{msec}, S E=13.5)$. One might speculate that this finding reflects incomplete or shallower processing for targets subsequent to arousing pictures, resulting in earlier LP peak latencies and smaller LP amplitudes. Future work may employ approaches such as independent component analysis to disentangle the differential contributions of stimulus processing, response selection, and response preparation in the current task.

Regional source modeling. Sensitivity maps of the regional dipoles (see Figure 8, left panel) demonstrated that the sources were spatially independent and reflected activity from nonoverlapping cortical areas. Across conditions, the model accounted for more than $96.8 \%$ of the variance in the grand-average global field power. As illustrated in the time-varying activity plots (Figure 8 , middle panel), source modeling yielded four activity peaks corresponding to picture P2, mask onset (see 
Figure 7. Experiment 1: Mean target LP amplitudes as a function of target type, picture category, and hemisphere. The LP was reduced for targets subsequent to emotional information. For word targets, LP reduction followed the U-shaped function of picture arousal ratings. This pattern was less pronounced for pseudoword responses. Error bars indicate standard errors.

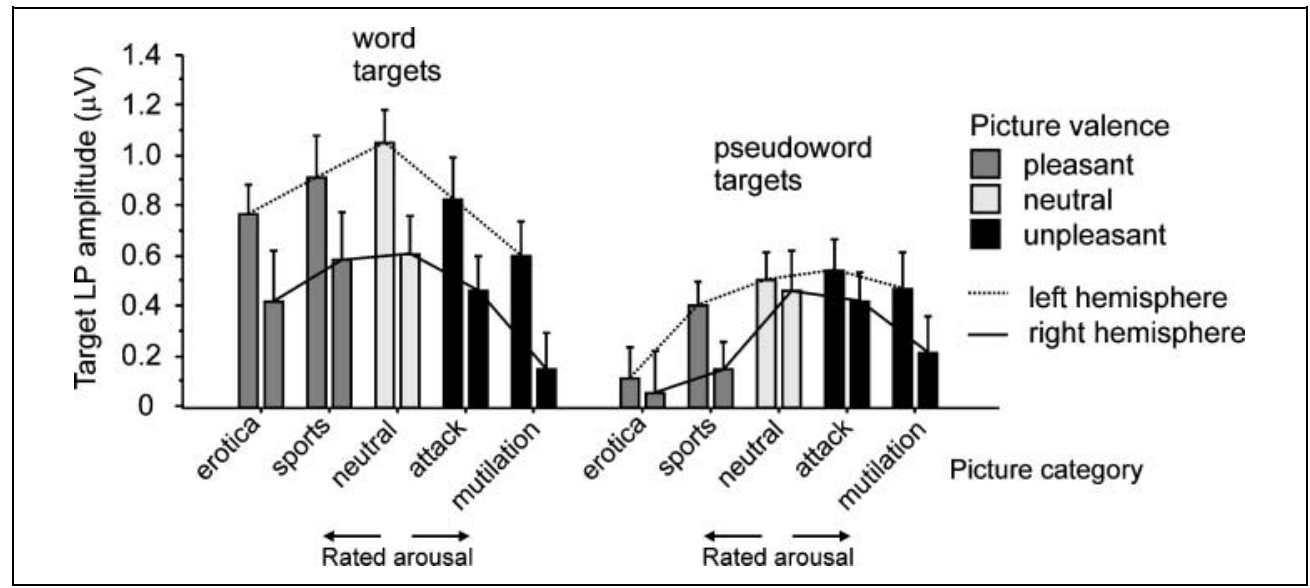

Electrophysiological Data), target N1, and target LP. The sources demonstrated substantial differences in their overall contribution to those activity peaks and a distinct pattern of intensity changes across time. For the picture P2 and target N1, the model was dominated by bilateral activity in the pIT with moderate contributions of occipital, aIT, parietal, and frontal sources. The target LP was associated with activity mainly originating from left sources in the aIT region and minor contributions of parietal and frontal sources. Central sources did not show major intensity changes pertinent to picture or target onset.

The question arises whether and where the source waveforms for different picture categories displayed a pattern similar to the condition differences in the scalp potentials. As can be seen in Figure 8, at the pIT, activity in the picture P2 window was more pronounced for neutral, compared to pleasant and unpleasant, pictures. Conclusions from this result must be drawn with caution. First, seeding of pIT sources aimed at modeling language-related activity in areas of lexico-semantic analysis (see Methods) and not at finding a best-fit model of early negativities. Second, prior to the P2 window, right pIT and occipital source waveforms displayed the expected activity amplification for arousing relative to neutral pictures. This may reflect that the early enhancement for emotionally arousing stimuli typically starts with an increase of the N1 component (Keil et al., 2001), and then continues as a relative negative shift within a positive-going deflection (the P2). Because of these dynamics, the EPN has been analyzed as a difference wave, subtracting emotionally arousing and neutral conditions (Schupp et al., 2003).

As described in the Methods section, effects of picture category (pleasant, neutral, unpleasant) on word/ pseudoword processing were statistically evaluated by calculating mean amplitudes in the target N1 (204$264 \mathrm{msec}$ ) and in the target LP (404-704 msec) time segment, for each source. In the early (N1) segment, a main effect of picture category emerged in the left
pIT (see right panel of Figure 8) $[F(2,42)=8.3, p<$ .001], reflecting reduced activity for words following pleasant and unpleasant pictures, compared to words following neutral exemplars (Newman-Keuls: $p s<.01$ ). For pseudoword targets, affective modulation in the $\mathrm{N} 1$ range was evident in the left pIT $[F(2,42)=6.5$, $p<.01]$, and at the left occipital source $[F(2,42)=5.6$, $p<.01]$. As for words, post hoc tests showed a reduction for pseudowords preceded by pleasant (left pIT: $p<.01$; left occipital: $p<.05)$ and unpleasant scenes (left pIT: $p<.05$; left occipital: $p<.01$ ), compared to neutral pictures. During this time window, the difference between targets following arousing versus nonarousing information did not reach the adjusted alpha level of $1 \%$ at any other source.

In the late (LP) time segment, significant effects of picture category were obtained for word targets in the left aIT $[F(2,42)=6.6, p<.01]$ and the left parietal cortex $[F(2,42)=6.6, p<.01]$. Relative to neutral pictures, source activity was reduced for words following pleasant $(p s<.05)$ and unpleasant scenes $(p s<.01)$. Source activity for pseudowords did not vary as a function of affective picture category.

\section{Experiment 2}

\section{Postexperimental Affective Ratings}

Picture category modulated valence ratings $[F(2,11)=$ 97.8, $p<.001]$ and arousal ratings $[F(2,11)=74.0$, $p<.001]$. Confirming that participants perceived the pictures as intended, pleasant pictures yielded higher valence scores (mean valence $=6.2, S D=1.2$; NewmanKeuls: $p<.05$ ), and unpleasant items obtained lower values (mean valence $=1.6, S D=0.3 ; p<.001$ ), compared to neutral stimuli (mean valence $=5.4, S D=1.0$ ). Both affective categories (pleasant: mean arousal $=4.8$, $S D=1.5$; unpleasant: mean arousal $=7.3, S D=0.9$ ) were rated as more arousing than the neutral category (mean arousal $=2.1, S D=1.2), p s<.001$. 


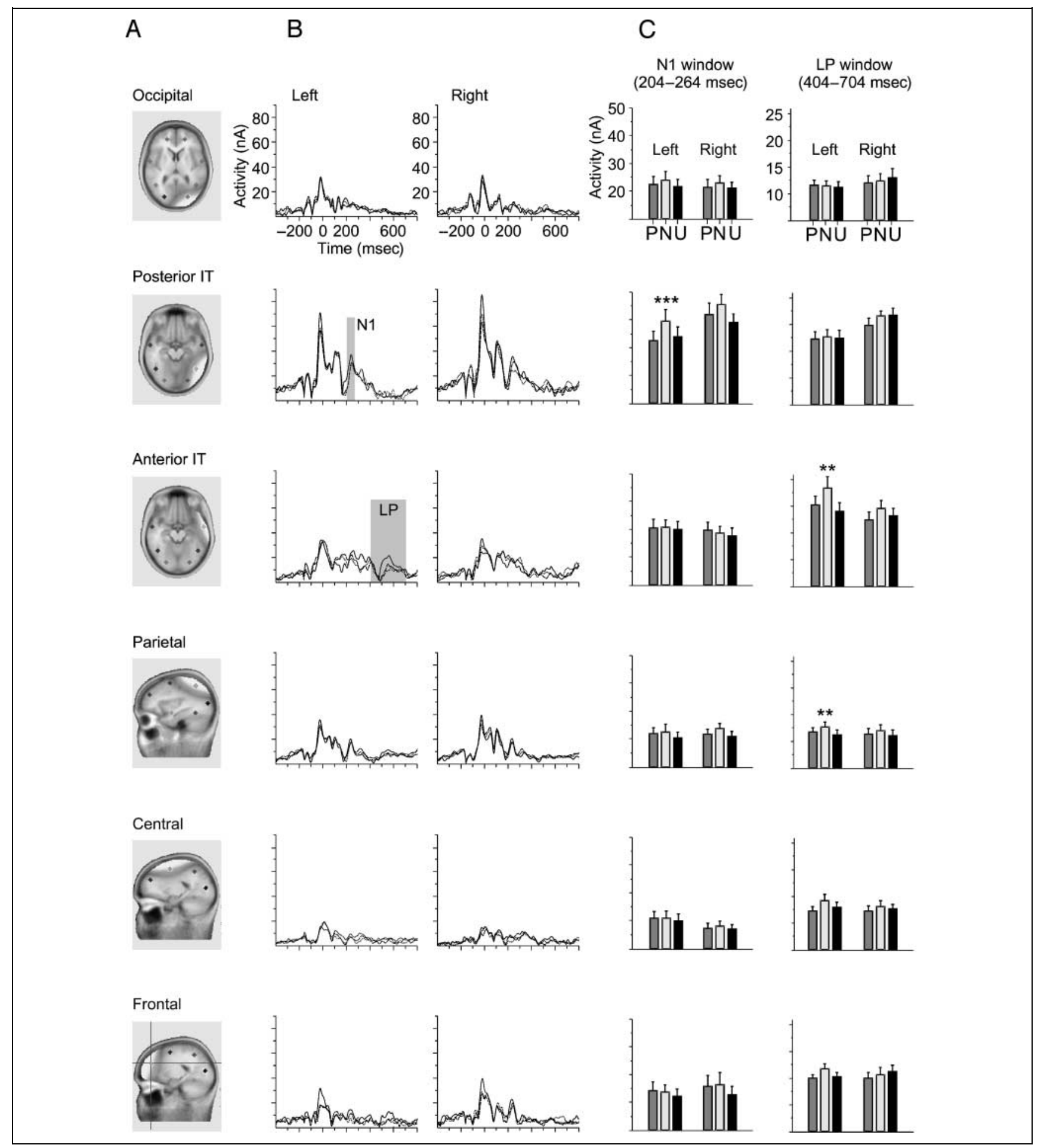

Figure 8. Experiment 1: Regional source modeling of word target ERPs. Each tier displays data of one cortical region, namely, bilateral symmetric sources in occipital, posterior inferior temporal (pIT), anterior inferior temporal (aIT), parietal, central, and frontal cortices. (A) Sensitivity maps of the regional sources illustrating the spatial independence of the used dipoles. Only maps of left hemispheric sources are shown. (B) Time-varying activity plots of source responses to words following pleasant (dashed line), neutral (solid line), and unpleasant pictures (dotted line). Source waveforms were obtained by applying the source montage to grand mean ERP data. Time zero represents word onset. (C) Mean source intensity in the $\mathrm{N} 1$ and LP time window as a function of picture category ( $\mathrm{P}=$ pleasant; $\mathrm{N}=$ neutral; $\mathrm{U}=$ unpleasant). Values were obtained by applying the source model to individual subjects' word ERPs and extracting mean activity in the respective time segment. In the N1 window, arousing pictures suppressed source activity in the left pIT. In the LP window, an effect of picture category was present in left aIT and parietal sources $(* * p<.001, * * p<.01)$. Error bars represent standard errors. 


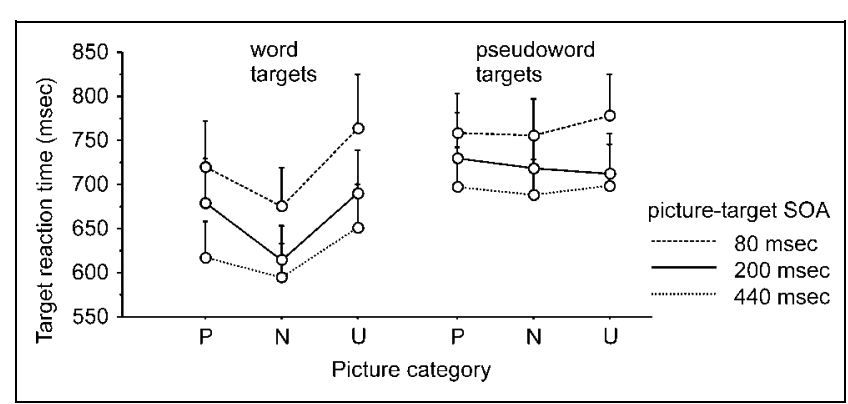

Figure 9. Experiment 2: Mean reaction times as a function of picture-target SOA, target type, and category of the preceding picture ( $\mathrm{P}=$ pleasant $\mathrm{N}=$ neutral; $\mathrm{U}=$ unpleasant $)$. At all SOAs, pleasant and unpleasant pictures impaired word, but not pseudoword, responses. Error bars indicate standard errors.

\section{Lexical Decision}

ANOVA on RTs yielded significant main effects of SOA $[F(2,22)=28.4]$ and target type $[F(1,11)=16.6, p s<$ $.01]$, and a significant interaction between both factors $[F(2,22)=4.1, p<.05]$. Post hoc tests showed that for both target types, RTs were faster at the short SOA (80 msec) and slower at the long SOA (440 msec), compared to the 200-msec SOA $(p s<.01)$. Importantly, we replicated the main effect of picture category $[F(2,22)=$ $10.5]$, and the interaction effect between picture category and target type $[F(2,22)=5.9, p<.01]$, while ANOVA did not show any significant interactions involving SOA and picture category. That is, at all SOAs, pleasant and unpleasant pictures elicited slower word responses than neutral pictures $(p s<.01)$, whereas emotional content did not modulate pseudoword responses $(p s>.49)$ (see Figure 9). These findings also suggest that the magnitude of interference exerted by erotica and mutilation pictures remains constant although task difficulty increases (indexed as an overall increase of RTs for shorter SOAs). ANOVA on error rates yielded a significant interaction between picture category and target type $[F(2,22)=11.1, p<.001]$. Across SOAs, unpleasant pictures, compared to neutral exemplars, provoked higher error rates during word trials $(p<.05)$. Errors on pseudoword trials were not modulated by picture category $(p s>.05)$.

The implications of Experiment 2 are twofold: (i) Affective interference as observed here is not a shortlived/transient phenomenon or a mere result of stronger sensory forward masking of emotional pictures. Otherwise, performance deficits should have vanished at the latest for the 400-msec SOA. Instead, erotica and mutilation pictures appear to trigger an affective response having sustained negative consequences for subsequent processing. This assumption is also supported by work from our laboratory showing persisting emotional interference for up to 1000 msec during processing of RSVP streams. (ii) Already shortly after the emotional picture, encoding of new information is impeded. The occur- rence of facilitation (e.g., Phelps et al., 2006) versus interference (e.g., Most et al., 2005) may thus not only be determined by the temporal interval between picture and target but by other task aspects, such as complexity or the degree of emotional arousal evoked by the lead stimuli.

\section{Experiment 3}

\section{Postexperimental Affective Ratings}

Ratings varied with picture category on both SAM dimensions [valence ratings: $F(4,14)=219.0$; arousal ratings: $F(4,14)=65.3 ; p s<.001]$. As intended, both $\mathrm{PH}$ (mean valence $=6.3, S D=1.0)$ and $\mathrm{PL}(7.1, S D=$ $0.7)$ pictures evoked higher pleasure scores than neutral items $(p s<.001)$. Similarly, UH $(1.5, S D=0.5)$ and UL (mean valence $=2.6, S D=0.6$ ) pictures both were related to lower unpleasantness, compared to neutral pictures $(p s<.001)$. For unpleasant items, subjectively rated arousal was higher for $\mathrm{UH}$ pictures (mean arousal $=$ 7.6, $S D=0.9)$, compared to UL (mean arousal $=5.7$, $S D=1.2)$ and NL (mean arousal $=2.8, S D=0.9)$ pictures $(p s<.001)$. PH pictures $(5.1, S D=1.6)$ were rated as more arousing compared to PL (mean arousal $=2.9, S D=$ 1.2) and NL items $(p s<.001)$.

\section{Lexical Decision}

Error rates were higher for pseudowords than words $[F(1,15)=17.1, p<.001]$, but not sensitive to influences of picture category $(F s<2.0)$. Again, pseudoword responses were also slower than word responses $[F(1,15)=17.1, p<.001]$ (Figure 10). Further, ANOVA on RTs yielded a main effect of picture category $[F(1,15)=$ $6.7, p<.001]$. The significant interaction Picture category $\times$

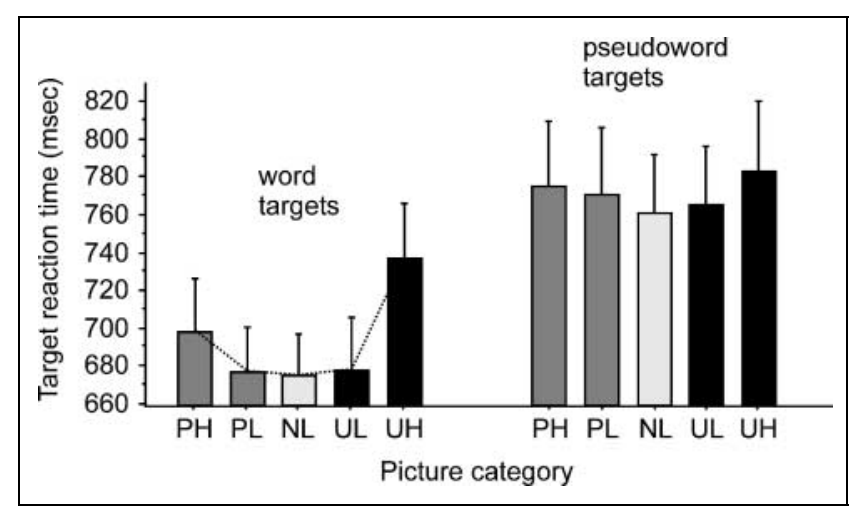

Figure 10. Experiment 3: Mean reaction times as a function of target type and category of the preceding picture $(\mathrm{PH}=$ high-arousing pleasant; $\mathrm{PL}=$ low-arousing pleasant; $\mathrm{NL}=$ low-arousing neutral; $\mathrm{UL}=$ low-arousing unpleasant; $\mathrm{UH}=$ high-arousing unpleasant). Word responses were delayed for high-arousing (pleasant and unpleasant) pictures but not for low-arousing items. Error bars indicate standard errors. 
Target type $[F(4,60)=2.7, p<.05]$ indicated that, similar to the previous experiments, picture category affected word and pseudoword processing differently. Although word responses showed a U-shaped interference pattern with most delayed RTs for the high-arousing categories (PH and $\mathrm{UH}$ ) [quadratic trend: $F(1,15)=$ 14.8, $p<.01$ ], modeling of pseudoword RTs did not yield a significant parabolic relationship $[F(1,15)=2.0$, $p=.17]$.

\section{Correlation Analyses}

On the level of individual subjects, the differences between arousal scores for emotional (PH, PL, UL, UH) versus neutral pictures significantly correlated with the respective differences of word RTs $(r=.33, p<.01)$. No such relationship was found for pseudowords $(r=.04$, $p=.74)$. Similarly, in an item-level correlation analysis calculated for single pictures, mean arousal scores given to pictures predicted mean RTs in the word $(r=.28$, $p<.001)$, but not in the pseudoword condition $(r=.12$, $p=.11)$.

Taken together, both ANOVA and correlation analyses indicate a parametric relationship between picture arousal and response impairment, specifically when processing word targets. Although pleasantness/unpleasantness was comparable for PL and PH pictures, and UL and UH pictures, respectively, only the high-arousing items impeded responses. Further, as picture categories were semantically heterogeneous (e.g., both PH and PL pictures comprised erotica), Experiment 3 thus provided strong evidence against the potential that RT interference in the present design is caused by salient semantic category differences.

\section{GENERAL DISCUSSION}

Optimized perceptual encoding of arousing versus nonarousing visual information is a pivotal finding in research on affective stimulus processing (e.g., Schupp et al., 2003; Anderson \& Phelps, 2001). In the present study, we aimed to track the consequences of such effects for a subsequent cognitive task. Verbal targets were presented after task-irrelevant picture stimuli, and participants executed a speeded lexical decision. Pictures varied in subjectively rated hedonic valence and emotional arousal.

At the level of motor output, we found delayed response latencies for word targets following high-arousing erotica and mutilation pictures at an SOA of $200 \mathrm{msec}$ (Experiment 1). By employing different SOAs, in Experiment 2 , the hypothesis was tested that behavioral interference versus facilitation is contingent on the temporal interval between picture and target. As a main result, emotionally arousing pictures interfered with lexical processing irrespective of SOA. Using the same SOA but different picture categories, Experiment 3 intended to disentangle the role of valence versus emotional intensity in the current paradigm. Emotional arousal now was varied within the aversive and appetitive categories, which included semantically mixed stimulus exemplars. We demonstrated that responses were impaired for pleasant and unpleasant pictures rated as highly arousing but not for affective exemplars related to low arousal scores. Again, interference was stronger for lexical targets.

At the level of electrocortical scalp potentials (Experiment 1), the N1-P2 complex to picture stimuli displayed a negative amplitude shift for arousing pictures, especially for erotica and mutilation scenes (cf., Schupp et al., 2003). With regard to target processing, those stimuli reduced N1 amplitudes and delayed N1 peak latencies both for word and pseudoword responses. Inhibitory effects of emotional content were demonstrated for the target LP as well. In contrast to the target N1, LP amplitudes were found to vary as a function of target type and picture category showing stronger impairment for word responses.

Taken together, ERP amplitudes and RTs in the present work were supportive of detrimental effects of affective stimuli on subsequent processing. Further, across experiments, the behavioral and electrocortical measures exploited here suggest interference as a function of emotional arousal. Facilitation as indicated by enhanced ERP components (Pourtois et al., 2004) or increased perceptual acuity (Phelps et al., 2006) has been recently reported for neutral information subsequent to emotional faces. Typically, judgments of emotional arousal are markedly lower for emotional faces compared to images depicting emotional scenes (Schupp, Öhman, et al., 2004). In addition to differences in experimental design, we propose that the emotional intensity of a preceding event is one crucial variable determining the occurrence of either facilitation or interference. This view is corroborated by the results of Experiment 3, which directly demonstrated that an increase of self-rated arousal is related to an increase of behavioral interference. Interference effects may result from resource-consuming analysis of emotionally arousing, complex visual scenes. In contrast, emotional faces may serve as salient cues for orienting attentional resources, exerting a facilitatory effect on subsequent processing. Future work may address the question how the complexity of the primary task, such as lexical decision versus orientation discrimination (Pourtois et al., 2004) or contrast detection (Phelps et al., 2006), mediates the divergent outcome of facilitation versus interference.

The gradual increase of interference with higher arousal scores is at variance with an alternative account drawing from theories on affective consistency. Faster RTs and larger N1 amplitudes for targets preceded by neutral versus emotional pictures may originate from 
facilitated processing for picture-target pairs having congruent hedonic valence (here, neutral-neutral vs. pleasant-neutral and unpleasant-neutral pairs). This assumption would predict that effects of picture subcategories having the same valence (e.g., sport scenes and erotica, see Methods) differ from effects of the neutral category to the same extent (Klauer \& Musch, 2003). This, however, was not observed in the present study.

\section{Target $\mathrm{N} 1$ and $\mathrm{LP}$ as Markers of Lexico-semantic Analysis}

Understanding emotional interference as observed here requires to consider possible functional roles of the target N1 and LP. A common finding in ERP studies on visual language processing pertains to a posterior negativity peaking around $200 \mathrm{msec}$ and differentiating orthographic (words, pseudowords, consonant strings) from nonorthographic (forms, symbols) stimuli (e.g., Bentin, Mouchetant-Rostaing, Giard, Echallier, \& Pernier, 1999). In a similar time segment, Cohen et al. (2000) reported a negativity over left inferior temporal sensors to words and consonant strings. Intracranial recordings from the posterior fusiform gyrus (Nobre, Allison, \& McCarthy, 1994) and brain imaging work (e.g., Petersen, Fox, Snyder, \& Raichle, 1990) have stimulated the idea that these early negative deflections to orthographic material may index processing within specialized visual word form systems of the inferior temporal lobe (Cohen et al., 2000).

The present results from regional source modeling are consistent with the concept of inferior temporal language functions. Target processing in the N1 time window (204-264 msec) was associated with predominant activity at the pIT sources, which we had seeded according to proposed coordinates of the visual word form area (Cohen \& Dehaene, 2004). We found pronounced pIT activity for both word and pseudoword targets. This supports the notion that the basal temporal cortex has a relative specificity for processing words and word-like stimuli (e.g., pseudowords), compared to random consonant strings or strings of nonalphabetic tokens (Petersen et al., 1990). However, our results neither corroborate the assumption that word form processing happens exclusively in the left pIT nor do they imply that the single function of the left posterior fusiform gyrus relates to word form processing (see also Price \& Devlin, 2003). As illustrated in Figure 8, pIT activation was substantial during picture processing. Moreover, activity during N1 target processing was comparable in size for the left pIT and the homologous area in the right hemisphere. Minor activations in the N1 window were also found in parietal and frontal sources.

We provide suggestive evidence that occipito-temporal processing around 200 to $250 \mathrm{msec}$ is not prelexical in nature as proposed by Dehaene, Le Clec, Poline, Le Bihan, and Cohen (2002). Specifically, the N1 amplitude was less negative to words than pseudowords. Lexicality effects in this latency range are a key finding of the recognition potential (RP) research (Martin-Loeches, Hinojosa, Gomez-Jarabo, \& Rubia, 2001). The RP has been referred to a bilateral negative deflection at parietooccipital sensors, typically $\mathrm{PO} 7$ and $\mathrm{PO} 8$, associated with conscious recognition of words or pictures (Rudell, 1992). Neuronal generators of the RP coincide with proposed locations of the visual word form area, namely, lingualis and/or fusiform gyrus in basal extrastriate areas (MartinLoeches et al., 2001). Interestingly, the RP has been linked to activation of left and right basal temporal cortices, which is consistent with our source modeling results showing bilateral pIT activation. In contrast to visual word form activity, the RP has been shown sensitive to the meaningfulness of the stimulus with responses gradually increasing from random letter strings to pseudowords and words (Martin-Loeches et al., 2001). According to this perspective, the $\mathrm{N} 1$ as found here might reflect processes related to lexical access (i.e., activation of entries in a hypothetical mental lexicon).

Lexicality effects on the target LP were reflected by a more negative positivity to pseudowords compared to words at centro-parietal sensors. This finding closely replicates those found in a forced-choice lexical decision paradigm by Hauk et al. (2006). It is also consistent with the N400 literature reporting increased negativity to pseudowords relative to words (Kutas \& Federmeier, 2000). Activity in the LP window mainly originated from left aIT, suggesting that stimulus analysis in the late time segment had progressed in the anterior direction. Within the left inferior temporal cortex, a directed processing stream proceeding anteriorly by continuous cortical processing has been demonstrated by Fernandez et al. (2001), who recorded intracranial ERPs during visual word processing. Using a similar methodology, Nobre et al. (1994) showed that anterior regions of the fusiform gyrus were sensitive to semantic stimulus dimensions, whereas posterior parts responded equally to words and nonwords. Semantic encoding in anterior temporal areas is also suggested by studies using positron emission tomography during visual word perception (Vandenberghe, Price, Wise, Josephs, \& Frackowiak, 1996). In the LP window, we also observed activity at left parietal and frontal sources, which points to semantic representation encompassing distributed activation in large-scale neuronal networks (Pulvermüller, 1999).

Taken together, the componentry as found here suggests continuous processing along the ventral stream and a corresponding gradual increase in the complexity or depth of analysis. N1 effects may mark the final stages of initial stimulus analysis, including lexical access, but also mediate the transition from surface/form analysis to content/lexico-semantic processing. LP effects, on the other hand, may index word processing on a postlexical level involving the formation of semantic associations 
and processes related to decision making and response planning (Hauk et al., 2006).

\section{Emotional Interference in Network Models of Stimulus Representation}

How do the proposed functions of target ERPs relate to the observed effects of emotional arousal? Source modeling showed that the pIT, particularly in the left hemisphere, constituted the dominant site of emotional interference effects in the N1 time segment. Paralleling the N1 scalp data, inhibition at pIT sources was present for both words and pseudowords. During the LP window, emotional interference was evident at the left aIT and parietal sources. Reduction effects, however, were limited to words. As indicated by RTs during pseudoword trials, interference in the early time segment (between 200 and $250 \mathrm{msec}$ ) does not necessarily manifest in overt response modulation. We speculate that a pseudoword decision can be achieved even if pIT activation is reduced to some amount. In contrast, word responses may be impaired because additional interference arises during later time windows (between 400 and $700 \mathrm{msec}$ ). Such postlexical processing after initial word form access in the pIT may be elicited automatically for words but not for pseudowords in more anterior regions of the ventral stream.

Another useful approach to explain emotional interference as observed here pertains to network models of stimulus representation (Anderson \& Bower, 1973). In this perspective, words differ from pseudowords in the size and cortical topography of the underlying representational network (Pulvermüller, 1999). Although pseudowords engage circumscribed cell assemblies representing physical, orthographic, and phonological properties, word representations additionally recruit connections to semantic memory comprising neurons in visual, motor, and multimodal association cortices. Activity in such large-scale semantic networks has recently been linked to centro-parietal ERPs showing a positive-going peak around 400 to $500 \mathrm{msec}$ (Hauk et al., 2006). In the same vein, the target LP displayed distributed centro-parietal topography and was more pronounced for words, compared to pseudowords. A comparison of source waveforms in this time segment revealed that source strength differences between words and pseudowords were present across several cortical regions. Words showed larger activity than pseudowords in right anterior temporal, right posterior temporal, and bilateral parietal sources. As indexed by shorter RTs to words compared to pseudowords, activation of semantic associations facilitated motor responses. At the same time, it made the cognitive system vulnerable to interference from ongoing affective processing. Such interference may be viewed in terms of neurophysiological suppression, which mediates the rivalry between temporally overlapping activation patterns (Keysers \&
Perrett, 2002). Supporting the notion of neuronal competition as a basis of emotional interference, perceptual networks representing affectively arousing stimuli have been characterized by strong and widespread connections to memories, as well as visceral and motor systems (Lang et al., 1997). As described earlier, enhanced late positive potentials to arousing pictures may indicate continued activity within such networks across a time range of several seconds (Cuthbert et al., 2000). The present results suggest that late interference in semantic networks determines the presence of emotional interference at the level of motor output. Inhibited activity in subsemantic network parts as observed for words and pseudowords in the N1 window may not be sufficient to modulate the speed of the manual response.

\section{Conclusion}

We conclude that processing emotionally arousing information interferes with lexico-semantic analysis of temporally adjacent signals. The observed effects of picture emotional intensity and target meaning may help to disentangle the controversy of affective processing, leading to either facilitated or impaired performance in a subsequent task. Emotional interference depends on the presence of highly arousing affective material. It increases with the amount of higher-order semantic encoding required for the primary task. Facilitatory effects, such as amplitude enhancement or response acceleration, may be specific to tasks with low perceptual complexity (e.g., orientation discrimination or contrast detection), which are presented subsequent to moderately arousing (face) stimuli. Further work is required to scrutinize the boundary conditions of emotion-induced facilitation versus interference for a given stimulus.

\section{Acknowledgments}

The present study was supported by grants from the German Research Council (Deutsche Forschungsgemeinschaft). We thank Nadine Stumpf, Hanna Gruell, and Christian Pietrek for help in data acquisition.

Reprint requests should be sent to Niklas Ihssen, Department of Psychology, University of Konstanz, P.O. Box D23, D-78457 Konstanz, Germany, or via e-mail: niklas.ihssen@unikonstanz.de.

\section{REFERENCES}

Anderson, A. K., \& Phelps, E. A. (2001). Lesions of the human amygdala impair enhanced perception of emotionally salient events. Nature, 411, 305-309.

Anderson, J. R., \& Bower, G. H. (1973). Human associative memory. Washington, DC: Winston.

Armony, J. L., \& Dolan, R. J. (2002). Modulation of spatial attention by fear-conditioned stimuli: An event-related fMRI study. Neuropsychologia, 40, 817-826.

Bentin, S., Mouchetant-Rostaing, Y., Giard, M. H., Echallier, J. F., \& Pernier, J. (1999). ERP manifestations of processing 
printed words at different psycholinguistic levels: Time course and scalp distribution. Journal of Cognitive Neuroscience, 11, 235-260.

Berg, P., \& Scherg, M. (1994a). A fast method for forward computation of multiple-shell spherical head models. Electroencephalography and Clinical Neurophysiology, 90, 58-64.

Berg, P., \& Scherg, M. (1994b). A multiple source approach to the correction of eye artifacts. Electroencephalography and Clinical Neurophysiology, 90, 229-241.

Binder, J. R., McKiernan, K. A., Parsons, M. E., Westbury, C. F., Possing, E. T., Kaufman, J. N., et al. (2003). Neural correlates of lexical access during visual word recognition. Journal of Cognitive Neuroscience, 15, 372-393.

Bradley, M. M., \& Lang, P. J. (1994). Measuring emotion: The Self-Assessment Manikin and the Semantic Differential. Journal of Behavior Therapy and Experimental Psychiatry, 25, 49-59.

Bradley, M. M., Sabatinelli, D., Lang, P. J., Fitzsimmons, J. R., King, W., \& Desai, P. (2003). Activation of the visual cortex in motivated attention. Behavioral Neuroscience, 117, 369-380.

Cacioppo, J. T., Crites, S. L., Jr., Berntson, G. G., \& Coles, M. G. H. (1993). If attitudes affect how stimuli are processed, should they not affect the event-related brain potential? Psychological Science, 4, 108-112.

Calvo, M. G., \& Castillo, M. D. (2005). Foveal vs. parafoveal attention-grabbing power of threat-related information. Experimental Psychology, 52, 150-162.

Cohen, L., \& Dehaene, S. (2004). Specialization within the ventral stream: The case for the visual word form area. Neuroimage, 22, 466-476.

Cohen, L., Dehaene, S., Naccache, L., Lehericy, S., Dehaene-Lambertz, G., Henaff, M. A., et al. (2000). The visual word form area: Spatial and temporal characterization of an initial stage of reading in normal subjects and posterior split-brain patients. Brain, 123 , 291-307.

Cuthbert, B. N., Schupp, H. T., Bradley, M., McManis, M., \& Lang, P. J. (1998). Probing affective pictures: Attended startle and tone probes. Psychophysiology, 35, 344-347.

Cuthbert, B. N., Schupp, H. T., Bradley, M. M., Birbaumer, N., \& Lang, P. J. (2000). Brain potentials in affective picture processing: Covariation with autonomic arousal and affective report. Biological Psychology, 52, 95-111.

Dehaene, S., Le Clec, H. G., Poline, J. B., Le Bihan, D., \& Cohen, L. (2002). The visual word form area: A prelexical representation of visual words in the fusiform gyrus. NeuroReport, 13, 321-325.

Fernandez, G., Heitkemper, P., Grunwald, T., Van Roost, D., Urbach, H., Pezer, N., et al. (2001). Inferior temporal stream for word processing with integrated mnemonic function. Human Brain Mapping, 14, 251-260.

Hauk, O., Davis, M. H., Ford, M., Pulvermüller, F., \& Marslen-Wilson, W. D. (2006). The time course of visual word recognition as revealed by linear regression analysis of ERP data. Neuroimage, 30, 1383-1400.

Hickok, G., \& Poeppel, D. (2004). Dorsal and ventral streams: A framework for understanding aspects of the functional anatomy of language. Cognition, 92, 67-99.

Hillyard, S. A., Vogel, E. K., \& Luck, S. J. (1998). Sensory gain control (amplification) as a mechanism of selective attention: Electrophysiological and neuroimaging evidence. Philosophical Transactions of the Royal Society of London, Series B, Biological Sciences, 353, $1257-1270$
Junghöfer, M., Bradley, M. M., Elbert, T. R., \& Lang, P. J. (2001). Fleeting images: A new look at early emotion discrimination. Psychophysiology, 38, 175-178.

Junghöfer, M., Sabatinelli, D., Bradley, M. M., Schupp, H. T., Elbert, T. R., \& Lang, P. J. (2006). Fleeting images: Rapid affect discrimination in the visual cortex. NeuroReport, 17, 225-229.

Keil, A., Bradley, M. M., Hauk, O., Rockstroh, B., Elbert, T., \& Lang, P. J. (2002). Large-scale neural correlates of affective picture processing. Psychophysiology, 39, 641-649.

Keil, A., \& Ihssen, N. (2004). Identification facilitation for emotionally arousing verbs during the attentional blink. Emotion, 4, 23-35.

Keil, A., Ihssen, N., \& Heim, S. (2006). Early cortical facilitation for emotionally arousing targets during the attentional blink. BMC Biology, 4, 23.

Keil, A., Müller, M. M., Gruber, T., Wienbruch, C., Stolarova, M., \& Elbert, T. (2001). Effects of emotional arousal in the cerebral hemispheres: A study of oscillatory brain activity and event-related potentials. Clinical Neurophysiology, 112, 2057-2068.

Keil, A., Stolarova, M., Heim, S., Gruber, T., \& Müller, M. M. (2003). Temporal stability of high-frequency brain oscillations in the human EEG. Brain Topography, 16, 101-110.

Keysers, C., \& Perrett, D. I. (2002). Visual masking and RSVP reveal neural competition. Trends in Cognitive Sciences, 6, $120-125$.

Klauer, K. C., \& Musch, J. (2003). Affective priming: Findings and theories. In J. Musch \& K. C. Klauer (Eds.), The psychology of evaluation: Affective processes in cognition and emotion (pp. 7-49). Mahwah, NJ: Erlbaum.

Kutas, M., \& Federmeier, K. D. (2000). Electrophysiology reveals semantic memory use in language comprehension. Trends in Cognitive Sciences, 4, 463-470.

Lang, P. J., Bradley, M. M., \& Cuthbert, B. N. (1997). Motivated attention: Affect, activation, and action. In P. J. Lang, R. F. Simons, \& M. T. Balaban (Eds.), Attention and orienting: Sensory and motivational processes (pp. 97-135). Hillsdale, NJ: Erlbaum.

Lang, P. J., Bradley, M. M., \& Cuthbert, B. N. (2005). International affective picture system (IAPS): Digitized photographs, instruction manual and affective ratings (Technical Report A-6). Gainesville, FL: University of Florida.

MacKay, D. G., Shafto, M., Taylor, J. K., Marian, D. E., Abrams, L., \& Dyer, J. R. (2004). Relations between emotion, memory, and attention: Evidence from taboo Stroop, lexical decision, and immediate memory tasks. Memory \& Cognition, 32, 474-488.

Martin-Loeches, M., Hinojosa, J. A., Gomez-Jarabo, G., \& Rubia, F. J. (2001). An early electrophysiological sign of semantic processing in basal extrastriate areas. Psychophysiology, 38, 114-124.

McCandliss, B. D., Cohen, L., \& Dehaene, S. (2003). The visual word form area: Expertise for reading in the fusiform gyrus. Trends in Cognitive Sciences, 7 , 293-299.

Micrografx. (1997). Graphics Suite 2: Micrografx.

Most, S. B., Chun, M. M., Widders, D. M., \& Zald, D. H. (2005). Attentional rubbernecking: Cognitive control and personality in emotion-induced blindness. Psychonomic Bulletin \& Review, 12, 654-661.

Nobre, A. C., Allison, T., \& McCarthy, G. (1994). Word recognition in the human inferior temporal lobe. Nature, 372, 260-263.

Öhman, A., Flykt, A., \& Lundqvist, D. (2000). Unconscious emotion: Evolutionary perspectives, psychophysiological 
data and neuropsychological mechanisms. In R. Lane \& L. Nadel (Eds.), The cognitive neuroscience of emotion (pp. 296-327). New York: Oxford University Press.

Oldfield, R. C. (1971). The assessment and analysis of handedness: The Edinburgh Inventory. Neuropsychologia, 9, 97-113.

Perrin, F., Pernier, J., Bertrand, O., \& Echallier, J. F. (1989). Spherical splines for scalp potential and current density mapping. Electroencephalography and Clinical Neurophysiology, 72, 184-187.

Petersen, S. E., Fox, P. T., Snyder, A. Z., \& Raichle, M. E. (1990). Activation of extrastriate and frontal cortical areas by visual words and word-like stimuli. Science, 249, 1041-1044.

Phelps, E. A., Ling, S., \& Carrasco, M. (2006). Emotion facilitates perception and potentiates the perceptual benefits of attention. Psychological Science, 17, 292-299.

Pourtois, G., Grandjean, D., Sander, D., \& Vuilleumier, P. (2004). Electrophysiological correlates of rapid spatial orienting towards fearful faces. Cerebral Cortex, 14, 619-633.

Pratto, F., \& John, O. P. (1991). Automatic vigilance: The attention-grabbing power of negative social information. Journal of Personality and Social Psychology, 61, 380-391.

Price, C. J., \& Devlin, J. T. (2003). The myth of the visual word form area. Neuroimage, 19, 473-481.

Pulvermüller, F. (1999). Words in the brain's language. Behavioral and Brain Sciences, 22, 253-279; discussion 280-336.

Ratcliff, R. (1993). Methods for dealing with reaction time outliers. Psychological Bulletin, 114, 510-532.

Rudell, A. P. (1992). Rapid stream stimulation and the recognition potential. Electroencephalography and Clinical Neurophysiology, 83, 77-82.
Sabatinelli, D., Bradley, M. M., Fitzsimmons, J. R., \& Lang, P. J. (2005). Parallel amygdala and inferotemporal activation reflect emotional intensity and fear relevance. Neuroimage, 24, 1265-1270.

Scherg, M., Ille, N., Bornfleth, H., \& Berg, P. (2002). Advanced tools for digital EEG review: Virtual source montages, whole-head mapping, correlation, and phase analysis. Journal of Clinical Neurophysiology, 19, 91-112.

Schupp, H. T., Cuthbert, B. N., Bradley, M. M., Hillman, H. H., Hamm, A. O., \& Lang, P. J. (2004). Brain processes in emotional perception: Motivated attention. Cognition and Emotion, 18, 593-611.

Schupp, H. T., Flaisch, T., Stockburger, J., \& Junghöfer, M. (2006). Emotion and attention: Event-related brain potential studies. Progress in Brain Research, 156, 31-51.

Schupp, H. T., Junghöfer, M., Weike, A. I., \& Hamm, A. O. (2003). Emotional facilitation of sensory processing in the visual cortex. Psychological Science, 14, 7-13.

Schupp, H. T., Öhman, A., Junghofer, M., Weike, A. I., Stockburger, J., \& Hamm, A. O. (2004). The facilitated processing of threatening faces: An ERP analysis. Emotion, 4, 189-200.

Ungerleider, L. G., \& Mishkin, M. (1982). Two cortical visual systems. In D. J. Ingle, M. A. Goodale, \& R. J. W. Mansfield (Eds.), Analysis of visual behavior (pp. 549-586). Cambridge: MIT Press.

Vandenberghe, R., Price, C., Wise, R., Josephs, O., \& Frackowiak, R. S. (1996). Functional anatomy of a common semantic system for words and pictures. Nature, 383, 254-256.

Zeelenberg, R., Wagenmakers, E. J., \& Rotteveel, M. (2006). The impact of emotion on perception: Bias or enhanced processing? Psychological Science, 17, 287-291. 